\title{
Droits de l'homme et disparitions
}

\section{Francis Perrin}

\section{(2) OpenEdition}

Journals

\section{Édition électronique}

URL : http://journals.openedition.org/conflits/175

DOI : 10.4000/conflits. 175

ISSN : $1777-5345$

Éditeur :

CCLS - Centre d'études sur les conflits lilberté et sécurité, L'Harmattan

\section{Édition imprimée}

Date de publication : 15 mai 1994

ISSN : 1157-996X

\section{Référence électronique}

Francis Perrin, «Droits de l'homme et disparitions », Cultures \& Conflits [En ligne], 13-14 I printemps-été 1994, mis en ligne le 14 mars 2006, consulté le 30 mars 2021. URL : http://journals.openedition.org/ conflits/175; DOI : https://doi.org/10.4000/conflits.175

Ce document a été généré automatiquement le 30 mars 2021.

Creative Commons License 


\title{
Droits de l'homme et disparitions
}

\author{
Francis Perrin
}

1 "Tout acte de disparition forcée constitue un outrage à la dignité humaine. Il est condamné comme un reniement des buts de la Charte des Nations Unies et comme une violation grave et flagrante des droits de l'homme et des libertés fondamentales proclamés dans la Déclaration Universelle des Droits de l'Homme et réaffirmés et développés dans les instruments internationaux dans ce domaine (...). Aucun Etat ne doit se livrer à des disparitions forcées, les autoriser ou les tolérer (...).Aucune circonstance, quelle qu'elle soit, qu'il s'agisse d'une menace de guerre, d'un état de guerre, d'instabilité politique intérieure ou de tout autre Etat d'exception, ne peut être invoquée pour justifier des disparitions". Ces citations sont extraites de la Déclaration sur la protection de toutes les personnes contre les disparitions forcées qui a été adoptée sans vote par l'Assemblée générale de l'Organisation des Nations Unies le 18 décembre $1992^{1}$. Elles soulignent le consensus officiel au sein de la communauté internationale visant à "contribuer par tous les moyens à prévenir et à éliminer les disparitions forcées" (art. 2). Si, dans le corps du texte, la Déclaration ne définit pas ce qu'est une disparition forcée, son préambule précise que, dans de nombreux pays, de telles violations des droits de l'homme existent "dans le sens où des personnes sont arrêtées, détenues ou enlevées contre leur volonté ou privées de leur liberté d'une autre manière par des agents de différents secteurs ou niveaux d'un gouvernement ou par des groupes organisés ou par des individus agissant pour le compte de ou avec le soutien, direct ou indirect, le consentement ou l'assentiment du gouvernement, situation suivie par un refus de révéler le sort et le lieu où se trouvent les personnes concernées ou un refus de reconnaître la privation de leur liberté, plaçant ainsi de telles personnes en dehors de la protection de la loi". Cette définition fait apparaître quatre éléments principaux : la privation de liberté; la responsabilité des agents de l'Etat ou de groupes ou de personnes agissant sous son contrôle ou avec son soutien ou, au minimum, avec son consentement; l'absence totale d'information sur le sort des victimes ; le déni de responsabilité de la part de l'Etat. Amnesty International parle de "disparitions" entre guillemets pour bien montrer que les victimes n'ont pas disparu volontairement. Quelqu'un sait ce qui leur est arrivé et quelqu'un est responsable de leur sort, qui est d'ailleurs souvent la mort. Mais tant que celle-ci n'a pas été confirmée 
(il s'agit alors d'une exécution extrajudiciaire ou d'un assassinat politique ${ }^{2}$ ), ou que les "disparus" n'ont pas été retrouvés vivants, AI continue à demander des explications sur le sort des "disparus". Dans cette contribution, nous voudrions aborder brièvement quatre points principaux pour tenter d'éclairer quelques aspects du phénomène politique que représentent les "disparitions" : L'évolution du contexte politique dans lequel se produisent les "disparitions". Les relations entre les "disparitions" et d'autres formes de répression politique et de violations des droits fondamentaux. Les liens entre l'efficacité des organisations non gouvernementales (ONG) de défense des droits de l'homme et l'émergence du phénomène des "disparitions". Les relations entre les "disparitions" et les exécutions extrajudiciaires.

2 Le contexte politique des disparitions "Les disparitions forcées se produisent généralement dans les pays où il existe de nombreuses tensions sociales et politiques et, à plus forte raison, dans ceux où les tensions tournent au conflit armé. Généralement, dans des circonstances de ce genre, la jouissance des droits de l'homme est mise à mal, le fonctionnement des institutions démocratiques affaibli et le respect des lois réduit à néant. Plus spécifiquement, il devient alors extrêmement difficile d'empêcher les disparitions forcées et d'enquêter à leur sujet. Les mécanismes destinés à protéger individuellement les citoyens en temps de paix s'avèrent vite futiles dans les conflits militaires, c'est-à-dire au moment où, précisément, une telle protection s'avère des plus nécessaires", note justement le groupe de travail des Nations Unies sur les disparitions forcées ou involontaires ${ }^{3}$. Au-delà de ce constat, il convient d'insister sur les modifications sensibles intervenues dans ce contexte politique au cours des trente dernières années. Dans les années 1960 puis dans les années 1970, les "disparitions" étaient essentiellement associées à des régimes militaires et à des dictatures. Cela est toujours le cas (Irak, Haïti) mais, au cours des deux décennies suivantes, la réalité s'est complexifiée et ce phénomène touche aujourd'hui d'autres pays dont la situation est très différente. Sans dresser une typologie exhaustive, les informations dont dispose Amnesty International permettent de mettre en lumière les autres catégories suivantes : Les régimes civils réunissant plusieurs des caractéristiques qui définissent une démocratie (pluralisme des partis politiques et des syndicats, garanties constitutionnelles et législatives, liberté de la presse, existence d'associations locales de défense des droits de l'homme, etc.) : Colombie, Inde et Philippines, par exemple. Les pays aux prises avec des conflits ethniques ou nationaux : ex-Yougoslavie, Soudan, exURSS. Les pays dans lesquels les forces de sécurité affrontent un ou des groupes armés d'opposition : Pérou, Sri Lanka, Turquie. Les pays traversant une grave crise politique : Zaïre. "Disparitions" et autres formes de répression La question est souvent posée de savoir si les "disparitions" sont une manifestation parmi d'autres (malgré de fortes spécificités) de la répression politique ou si, de par les avantages qu'elles présentent pour les gouvernements, elles constituent en quelque sorte une forme "supérieure" de la terreur d'Etat. Dans la première hypothèse, les "disparitions" viendraient s'ajouter à de nombreuses autres violations des droits de l'homme. Dans la deuxième hypothèse, par contre, elles se substitueraient à d'autres formes de répression considérées à tort ou à raison comme moins "efficaces". L'examen de la réalité actuelle ne permet pas de retenir globalement la deuxième hypothèse même s'il est indéniable que, dans le passé, plusieurs gouvernements ont accordé la priorité à une politique systématique de disparitions forcées, l'Argentine de la fin des années 1970 en constituant l'un des exemples les plus tristement célèbres. Depuis le début des années 1990 et dans les pays qui les pratiquent, les "disparitions" coexistent avec d'autres violations, comme le 
montre la liste suivante préparée à partir du dernier rapport annuel d'Amnesty International portant sur l'année $1992^{4}$.

Principales violations des droits de l'homme relevant des objectifs d'Amnesty ${ }^{5}$

4 Burundi Indonésie et Timor

5 "Disparitions" "Disparitions"

$6 \quad \mathrm{EEJ}{ }^{6} \mathrm{EEJ}$

7 Prisonniers d'opinion Prisonniers d'opinion

8 Procès inéquitables Procès inéquitables

9 Torture Détentions sans inculpation ni jugement

10 Traitements cruels, inhumains et dégradants Torture et mauvais traitements

11 Exécution capitale

12 Colombie Irak

"Disparitions" "Disparitions"

EEJ EEJ

Torture Prisonniers d'opinion

Torture

Exécutions capitales

Guatemala Liban

9 "Disparitions" "Disparitions"

EEJ EEJ

Torture et mauvais traitements Torture et mauvais traitements

2 Haïti Mauritanie

3 "Disparitions" "Disparitions"

24 EEJ EEJ

5 Prisonniers d'opinion Prisonniers d'opinion

Torture Torture

Inde Papouasie-Nouvelle Guinée

"Disparitions" "Disparitions"

EEJ EEJ

Prisonniers d'opinion Torture et mauvais traitements

Détentions sans inculpation ni jugement,

détentions au secret

Torture et mauvais traitements

Exécutions capitales

Pérou Tchad

"Disparitions" "Disparitions"

EEJ EEJ

Procès inéquitables Prisonniers d'opinion

Torture et mauvais traitements Torture et mauvais traitements

Cultures \& Conflits, 13-14 | printemps-été 1994 

répression des Etats aux efforts des ONG telles qu'Amnesty International s'inscrit dans l'hypothèse de substitution évoquée ci-dessus. Nous avons vu qu'elle n'était d'ailleurs pas la plus pertinente pour analyser la situation actuelle. Dans un sens sans doute plus polémique mais stimulant, certains mettent en cause la responsabilité du "lobby des droits de l'homme" dans l'émergence du phénomène des disparitions. "Il existe un sentiment de malaise au sujet du fait que les succès mêmes du mouvement des droits de l'homme en organisant des campagnes internationales poussent les gouvernements répressifs non pas à respecter les droits de leurs citoyens mais vers des formes de répression toujours plus horrifiantes. Il est devenu plus facile pour les gouvernements de faire disparaître leurs critiques plutôt que de risquer une publicité embarrassante en les détenant prisonniers", écrit ainsi Carline Moorehead ${ }^{7}$ qui se demande où sont passés les prisonniers d'opinion ${ }^{8}$. Dans sa réponse, Amnesty International a souligné que, malheureusement, les prisonniers d'opinion sont encore fort nombreux (Cuba, Pérou, Chine, Indonésie, etc.) à travers le monde aujourd'hui. Par ailleurs, la relation causale proposée pour expliquer les politiques de "disparitions" est beaucoup trop simpliste pour rendre compte de la complexité du phénomène. Amnesty ne peut, pour sa part, avancer un seul cas dans lequel l'adoption par un gouvernement de cette méthode de répression pourrait être clairement reliée au travail des ONG en faveur des prisonniers. Ainsi, lorsqu'à la fin des années 1960 le Guatemala a quasiment "remplacé" l'emprisonnement à caractère politique par les disparitions et les assassinats, ce pays n'était pas du tout dans la ligne de mire des mouvements des droits de l'homme ou de

Philippines Thaïlande

EEJ Torture et mauvais traitements

Prisonniers d'opinion Prisonniers d'opinion

Soudan Turquie

"Disparitions" "Disparitions"

Prisonniers d'opinion Prisonniers d'opinion

Torture Torture

Flagellation

Sri Lanka Zaïre

"Disparitions" "Disparitions"

Assassinats politiques EEJ

Torture et mauvais traitements Prisonniers d'opinion

Détentions sans inculpation ni jugement Procès inéquitables

Détentions sans inculpation ni jugement

Les "disparitions" : adaptation des Etats face au travail des ONG de défense des droits de l'homme? 
la communauté internationale 9 . De plus, les "disparitions" ne sont pas un phénomène nouveau. Pour ne remonter qu'à la deuxième guerre mondiale, l'historien François Bédarida a bien décrit la procédure "Nuit et Brouillard" inventée par le IIIème Reich pour "codifier et réglementer la disparition systématique de milliers d'adversaires de l'Allemagne national - socialiste" ${ }^{10}$. L'impression de croissance récente du phénomène n'est-elle pas surtout le résultat du développement des mouvements de défense des droits de l'homme, de leur capacité de recherche et d'une plus grande et rapide circulation de l'information, toutes choses qui font que notre connaissance des violations des droits de l'homme dans le monde, bien que partielle, est beaucoup plus étendue qu'elle ne l'était il y a 10, 20 ou 30 ans?

"Disparitions" et exécutions extrajudiciaires "Disparitions" et EEJ sont très fréquemment liées. De nombreux pays les pratiquent ou les ont pratiquées et la plus grande partie des premières sont suivies par des assassinats. Cependant, au moins au cours des dernières années, on constate que la fréquence des EEJ est plus importante que celle des "disparitions". Depuis le début 1990, Amnesty International a eu connaissance de "disparitions" dans plus de 30 pays. Or, au cours de la même période, des EEJ auraient eu lieu dans plus de 60 pays.

Pour l'année 1992 et en plus des Etats déjà listés dans notre deuxième partie, des EEJ ont été commises dans les pays suivants :

\section{Afghanistan Cambodge Israël Pakistan}

Afrique du Sud Comores Jamaïque Rwanda

Algérie Croatie Lesotho Sénégal

Angola Djibouti Liberia Sierra Leone

Azerbaïdjan El Salvador Mali Somalie

Bangladesh Ethiopie Moldavie Tadjikistan

Bosnie Géorgie Myanmar Togo

Brésil Iran Ouganda Venezuela

Yémen

Source : Amnesty International.

Bien que l'on ne dispose pas de mesure précise du phénomène et que la prudence soit de rigueur, il est probable que les "disparitions" soient globalement en recul par rapport aux années 1970 et 1980. C'est ainsi la situation de plusieurs pays d'Amérique Latine (Argentine, Chili, Salvador) ou d'Afrique (Ouganda, Namibie et, plus récemment, Maroc). Le cas du Maroc est à cet égard exemplaire. Utilisées pendant près d'une trentaine d'années depuis le début des années 60, les disparitions forcées ont été abandonnées au début de cette décennie sous l'effet de pressions internes (existence d'ONG locales) et externes (dont celles d'Amnesty International) ${ }^{11}$. Tout en concluant sur ce constat prudent, la complexité du phénomène des "disparitions" et l'imprévisibilité des changements politiques nous conduit à ne pas extrapoler les tendances récentes qui soulignent cependant qu'en matière de droits de l'homme il n'y a pas de fatalité. Mais, pour les pays qui ont renoncé à cette politique, il reste encore à accomplir un double devoir de vérité et de justice afin d'établir les responsabilités et de sanctionner les coupables. Et, sur ces deux points, l'expérience montre que bien peu d'Etats ont eu le courage politique d'affronter le passé. 

marquants de ces dernières années est l'émergence sur la scène internationale d'un grand nombre de groupes d'opposition armés qui ont commis et commettent encore de très graves exactions. Pour en rester aux thèmes qui font partie du domaine d'action d'Amnesty International, on peut citer la détention de prisonniers d'opinion, les prises d'otages, les mauvais traitements, mutilations et tortures, les exécutions parfois précédées de "procès", les assassinats politiques ou les déplacements forcés de population. Le lecteur pourra s'étonner de l'absence dans cette liste des mots disparitions forcées. Mais il ne s'agit pas d'un oubli. A quelques exceptions près, Amnesty International n'a pas d'informations sur des "disparitions" par de tels groupes. ${ }^{12}$. La question qui vient immédiatement à l'esprit est : pourquoi ? Quelles sont les spécificités de ce phénomène qui expliquent cette absence dans une liste malheureusement fort bien fournie à travers le monde ? Le problème est complexe et nous ne ferons ici qu'évoquer quelques pistes, pas toutes compatibles entre elles d'ailleurs. droits de l'homme, l'information dont on dispose est toujours partielle. Elle n'est que très rarement donnée mais le plus souvent "arrachée" dans des conditions difficiles et toute appréciation globale doit tenir compte de ce contexte. L'absence d'informations n'équivaut pas forcément à l'inexistence d'une violation. Pendant longtemps, de nombreuses organisations de défense des droits de l'homme ont concentré leurs efforts sur les gouvernements et c'est aussi le cas d'Amnesty. Cette exclusivité ou cette priorité se justifie par deux raisons de droit et une raison de fait. Premièrement, ce sont les gouvernements et non des groupes armés qui ont le devoir, au sens juridique sinon moral, de garantir la vie, la sécurité et les libertés de leurs ressortissants. C'est même la clé de leur légitimité. Deuxièmement, ce sont les Etats qui contractent des engagements internationaux de nature contraignante en ratifiant ou en adhérant à des pactes, des conventions ou des traités et non des groupes armés ${ }^{13}$. Troisièmement, les Etats sont responsables de la grande majorité des graves atteintes aux droits de l'homme et, lorsqu'ils en sont les auteurs, leurs victimes ne disposent d'aucun recours. Ainsi, jusqu'en 1991, Amnesty International ne condamnait que la torture et l'exécution de prisonniers par les groupes d'opposition armés. Cette année-là, au Conseil International de Yokohama (Japon), la plus haute instance de l'organisation, Amnesty décidait d'accroître de façon très sensible son travail dans ce domaine en incluant les prises d'otages et les "meurtres délibérés et arbitraires" commis par de tels groupes, quelle que soit leur importance, à condition que ces groupes soient à caractère politique ${ }^{14}$. $\mathrm{Ce}$ n'est donc que très récemment qu'Amnesty International a développé son effort de recherche sur les exactions des groupes politiques armés non liés à des gouvernements. Dans le mouvement mondial des droits de l'homme, d'autres organisations ont suivi la même évolution, d'autres l'ont quelque peu précédée et certaines continuent à ne s'intéresser qu'aux seuls gouvernements. Le champ d'investigation est donc largement nouveau.

La complexité organisationnelle des "disparitions" Dans un arrêt datant de juillet 1988, la Cour Interaméricaine des Droits de l'Homme avait souligné la complexité des "disparitions" parmi d'autres violations des droits de l'homme. De fait, une "disparition" implique un grand degré de dissimulation. Après l'arrestation ou l'enlèvement, le plus souvent de façon discrète, il faut cacher une personne dans un 
centre secret de détention ou se débarrasser de son cadavre. Il faut, surtout, tenter d'effacer toutes les preuves ou les éléments de preuve qui permettraient de remonter la chaîne des responsabilités. Cela suppose souvent d'intimider, de terroriser ou de liquider physiquement des témoins gênants (ou de les faire eux aussi disparaître), de ne pas laisser derrière soi d'indices matériels, de brouiller les pistes et de mener une véritable politique de désinformation, de s'opposer à l'ouverture d'enquêtes indépendantes, administratives ou judiciaires, ou de pratiquer des manoeuvres d'obstruction, etc... De telles conditions supposent fréquemment l'implication de l'appareil d'Etat, au minimum de certains secteurs des forces de sécurité. Les groupes armés d'opposition ne peuvent pas forcément maîtriser la complexité organisationnelle de ce phénomène. Les groupes armés n'ont pas de manière générale, le même souci de respectabilité que des gouvernements. Or, l'un des grands avantages des disparitions forcées qui "réussissent" est qu'elles permettent au pouvoir politique de nier sa responsabilité directe ou indirecte dans de très graves violations des droits de l'homme et, donc, de préserver une certaine image de marque interne et/ou internationale que viendraient ternir la détention de prisonniers d'opinion, de nombreux procès, des tortures ou des exécutions capitales. La dissimulation que permettent les "disparitions" accompagne souvent une répression "sophistiquée" pour des besoins d'image dont beaucoup de groupe armés n'ont cure. Loin de cette respectabilité, les groupes armés entendent donner la plus grande publicité à leurs actes afin de faire parler d'eux, de prouver que l'Etat qu'ils combattent ne contrôle pas son territoire ou est impuissant à protéger sa population, d'établir un rapport de forces politico-militaire qui leur soit favorable, etc. De là peut venir la très grande fréquence des assassinats politiques par ces groupes dont certains ont tué des milliers ou des dizaines de milliers de personnes (Sentier Lumineux au Pérou, Résistance Nationale du Mozambique, groupes séparatistes au Pendjab indien ou milices libanaises, par exemple). Ce rapport de forces qui, dans la plupart des cas, penche en faveur des gouvernements explique sans doute aussi la fréquence des prises d'otages par des groupes tels que les Forces Armées Révolutionnaires de Colombie, l'Armée de Libération Nationale (Colombie), les Tigres de Libération de l'Eelam Tamoul (Sri Lanka) et d'autres organisations. Ces prises d'otages peuvent avoir des objectifs financier, médiatique, politique qui peuvent d'ailleurs se cumuler et visent globalement à arracher des concessions à des Etats.

"Disparitions" et entités quasi-gouvernementales Ces différentes pistes doivent-elles nous conduire à conclure que les "disparitions" sont un mode de répression réservé aux gouvernements ${ }^{15}$ ? Bien que l'argumentation esquissée ici puisse le laisser penser, la conclusion nous semble trop hâtive. Le manque d'informations précises sur les activités des groupes politiques armés doit se traduire par une grande prudence dans ce domaine. De plus, la situation des droits de l'homme est extrêmement mouvante et l'avenir ne peut être figé. Enfin, si les spécificités respectives des gouvernements et des groupes d'opposition armés sont à prendre en compte, peut-on exclure que, parmi l'ensemble de ces groupes, ceux qui présentent les caractéristiques les plus proches de celles d'un gouvernement puissent à la fois maîtriser la complexité du processus des "disparitions", rechercher une certaine respectabilité et une apparence de légalité et utiliser les "disparitions" comme instrument de pouvoir politique sur le territoire et/ ou la population qu'ils contrôlent?

ANNEXE

LE MAROC ${ }^{16}$ 
80 Le problème sahraoui Il est difficile de parler des "disparus" sahraouis, car au Maroc personne n'osait et n'ose, même encore maintenant, véritablement l'aborder. La fameuse "marche verte" en 1975 a endormi les consciences des Marocains qui se réfèrent toujours à l'interdiction de porter atteinte à l'intégrité territoriale. On peut observer qu'encore le 30 juin dernier, les partis d'opposition n'étaient toujours pas prêts à reconnaître une éventuelle indépendance de la République sahraouie puisqu'on pouvait voir dans une information de l'AFP que "quatre partis marocains d'opposition ont adressé au Président égyptien, M. Hosni Moubarak, nouveau président de l'OUA, un message lui demandant de suspendre l'adhésion de la prétendue République sahraouie de l'organisation panafricaine...." "celle-ci ne disposant au Sahara Occidental d'aucune entité territoriale ou populaire pas plus que de légalité internationale. Et pourtant, Amnesty a estimé qu'entre 1975 et 1985, plusieurs centaines de personnes du Sud marocain et du Sahara occidental ont été arrêtées par des forces de sécurité et ont disparu dans des centres secrets. Bien que les Sahraouis instruits aient été plutôt la cible des "disparitions", des vieillards et de tous jeunes enfants figurent au nombre des victimes. Même des familles entières "disparaissaient". Presque tous les sahraouis maintenus en détention prolongée sans jugement pour des motifs politiques n'ont jamais bénéficié des procédures légales normales. Dans tous les cas, les informations recueillies laissent à penser que les autorités marocaines soupçonnaient les victimes ou leurs familles de soutenir le Front Polisario. C'est ainsi que Ahmed Lemaadal Mohamed Medhi El Bou, originaire de Smara, une des principales villes du Sahara occidental, a disparu à 33 ans, après avoir été arrêté le 15 avril 1976 au cours d'une rafle de civils sahraouis soupçonnés de soutenir le Front Polisario. Il avait servi dans l'armée espagnole, et comme la plupart des autres militaires d'origine sahraouie, avait été rendu à la vie civile à la veille de l'invasion de la colonie espagnole par le Maroc. Sa femme et ses trois fils sont sans nouvelles de lui. Ils ont fui le Sahara occidental pour rejoindre les milliers de réfugiés sahraouis des camps du Front Polisario dans le sud de l'Algérie. Ainsi le Sahraoui Kenti Sidi Balla peut témoigner de ce qu'il a vécu: "Je fus arrêté le 19 juin 1987 par des soldats marocains... ils m'ont remis à la gendarmerie royale... là j'ai été torturé et interrogé pendant 24 heures... j'ai été transféré dans un autre centre de détention à Skoura où il y avait quatorze autre Sahraouis dont des femmes... Au bout de deux ans j'ai été transféré au centre secret de Kalaat M'Gouna. Il fut libéré en août 1991.

81 Arbitraire et vengeance La multiplicité des systèmes policiers fait qu'il était souvent difficile de savoir pourquoi certains opposants plutôt que d'autres "disparaissaient". Le choix pouvait être totalement arbitraire. Mohamed Nadrani en est l'exemple type. En 1976 Mohamed Nadrani avait 22 ans, il était étudiant et était syndiqué comme beaucoup d'autres étudiants. Certains de ses camarades ont été arrêtés et emprisonnés, d'autres relâchés, lui a été maintenu en détention secrète pendant neuf ans, dont dix huit mois à l'isolement total. Après sa libération, il a demandé à consulter son casier judiciaire. Il était vierge. Officiellement, Mohamed Nadrani n'a jamais été détenu.

82 Cependant, les "disparitions" étaient aussi dues à des vengeances personnelles. Une personne commettant un délit pouvait provoquer la "disparition" d'un membre de la famille ou de la famille tout entière. Donnons deux exemples pour illustrer ce type de "disparition".

83 La famille Oufkir Huit membres de la famille ont été détenus pendant 18 ans, simplement à cause de leurs liens familiaux avec le général Mohamed Oufkir, décédé 
dans des circonstances mystérieuses après qu'il ait mené un coup d'Etat contre le roi. Sa veuve, ses six enfants, dont le plus jeune n'avait que 3 ans, ainsi qu'une cousine avaient subitement disparus. Les autorités marocaines ne fournissaient aucune information sur leur sort et leur lieu de détention, comme s'ils n'avaient jamais existé. C'est parce qu'en 1987, quatre enfants ont réussi à s'évader, rencontré un avocat français qui a alerté l'opinion publique que la famille sera enfin libérée en 1991. Le pouvoir s'acharne encore sur cette famille innocente, puisqu'elle reste interdite de passeport, malgré une pression nationale et internationale importante.

Les militaires de Tazmamart Cinquante huit militaires qui avaient participé à un attentat contre le roi et qui avaient été jugés et condamnés, pour la plupart, à des peines allant de 3 ans à 10 ans de prison, ont été transférés de la prison centrale de Kénitra au centre secret de Tazmamart, dans les contreforts de l'atlas. Ce centre avait été spécialement construit pour eux. Les cinquante huit cellules n'avaient ni fenêtre, ni éclairage, suffocantes l'été et glaciales l'hiver. Pendant 18 ans, ils ne sont jamais sortis de leurs cellules, n'ont jamais vu le soleil et n'ont jamais reçu aucun soin médical. Deux ans après leur arrivée à Tazmamart, deux d'entre eux terminèrent de purger leur peine. Voyant qu'ils n'étaient pas libérés, comme ils s'y attendaient, l'un d'eux se plaignit au gardien : "Tu en avais pour combien ?" il répondit "Trois ans". Le gardien rétorque : "Ne dis pas trois ans, dis la perpétuité". Vingt huit prisonniers moururent à Tazmamart. Seuls trente d'entre eux furent libérés en 1991.

La raison d'Etat, l'opposition politique au régime n'étaient pas les seules causes de "disparitions". Il pouvait aussi y avoir des jalousies privées, des rivalités personnelles ou des opérations financières secrètes. C'est ainsi que l'on peut citer le cas incroyable des trois frères Bourequat, de nationalité française et proche du Makzen (pouvoir royal). Ils furent enfermés de 1973 à 1991, dont les dix dernières années à Tazmamart, sans inculpation, ni jugement. Dans leurs témoignages, ils ont affirmé que "leur affaire avait commencé par la dénonciation au roi, sa majesté Hassan II, d'une conspiration dirigée par le chef des services secrets marocains, le colonel Dlimi".

En finir avec les disparus A l'heure actuelle, bien qu'il y ait encore environ 80 "disparus" marocains et 485 "disparus" au Sahara occidental, on ne disparaît plus, ou presque plus, au Maroc. La diminution du nombre des "disparitions" signalées depuis 1987 peut être due à l'amélioration du respect des limites légales de la garde à vue. En effet, le 30 décembre 1991, le roi Hassan II a promulgué une nouvelle loi amendant certains articles du code de procédure pénale marocain relatifs à la garde à vue (période pendant laquelle la personne interpellée est retenue par la police sans pouvoir entrer en contact avec sa famille ou un avocat).

Cependant d'autres raisons peuvent être avancées :

88

- Il semble que le pouvoir se sente plutôt embarrassé, en fait, on pourrait même dire pris à son propre piège. Il nie un jour, libère le lendemain, exige qu'on n'en parle plus, menace même. Il tergiverse et se rend à l'évidence malgré lui. Rappelons-nous: En novembre 1990, le Comité des Droits de l'Homme des Nations Unies ayant soulevé enfin le problème de Tazmamart, les représentants marocains avaient alors déclaré que le nom de Tazmamart ne figurait sur aucune des listes officielles des prisons. En juillet 1991, le roi Hassan II affirmait publiquement que "Tazmamart n'existait que dans l'esprit et l'imagination de personnes mal intentionnées". Un mois plus tard, les 28 militaires qui avaient survécu sortaient de leur bagne-mouroir. Et ce n'était qu'en 1992 
que le roi reconnaissait l'existence de Tazmamart. Il déclarait alors pour le journal Libération qu'"il s'agissait d'un lieu où ont été gardées des personnes qui y ont été administrativement assignées. Il n'a plus de raison d'être. Ce chapitre est clos. Il y a eu. Il n'y a plus. C'est tout".

- Autre raison : le livre de Gilles Perrault "Notre ami le roi", qui avait fait grand bruit et peut-être atteint quelques consciences endormies, bien que le pouvoir ait essayé d'en arrêter la diffusion au Maroc.

- N'oublions pas aussi le travail incessant de nombreuses associations de droits de l'homme en dehors du Maroc ainsi bien sûr qu'une grande campagne au niveau international organisée par Amnesty en 1991. Toutes ces manifestations ont eu pour effet, en 1991, la libération de plus de 260 Sahraouis, de 28 militaires de Tazmamart, des trois frères Bourequat et de la famille Oufkir. Bien que, depuis 1991, on soit obligé de constater, qu'au strict point de vue des libérations de "disparus", nous n'avons obtenu aucun résultat, cependant nous pouvons dire que maintenant le Maroc est un pays avec des forces intérieures puissantes demandant le changement et avec un gouvernement sensible à son image et aux pressions extérieures. En effet :

- Les journaux d'opposition n'hésitent plus à dénoncer les arrestations et les jugements inéquitables et surtout, ce qui est nouveau, demandent avec insistance que tous les "disparus soient libérés". Ils osent publier des lettres ouvertes d'anciens prisonniers de Tazmamart qui demandent réparation aux autorités. Et même, ils font paraître des interview de familles de disparus comme celle par exemple des parents de Houcine el Manouzi : Ce syndicaliste avait 28 ans lorsqu'il a été condamné à mort par contumace, à Marrakech, en septembre 1971, il a été enlevé à Tunis, l'année suivante, le 28 octobre 1972, et ramené au Maroc. Selon les observateurs d'Amnesty International, le procès avait été manifestement inéquitable, la majorité des accusés ne pouvant avoir commis les crimes qui leur étaient reprochés. Depuis 21 ans sa famille n'a rien pu savoir de lui, sinon qu'il a tenté de s'évader d'un lieu de détention avec d'autres "disparus". Il semble avoir été repris une semaine plus tard. Sa famille habite Paris et a créé l'Association des Parents et Amis des Disparus au Maroc.

- Et puis maintenant les langues se délient. Les anciens "disparus" crient bien haut qu'il faut parler, qu'il faut témoigner, que le mur du silence doit tomber.

- L'Association des Familles des Disparus et des Prisonniers politiques s'est mobilisée le 10 décembre dernier, à l'occasion du 45 ème anniversaire de la Déclaration Universelle des Droits de l'Homme, pour demander la libération de tous les "disparus" et les prisonniers politiques. Ce même jour, deux organisations des Droits de l'Homme exigeaient que la lumière soit faite sur les prisonniers politiques "disparus". Elles n'hésitent plus à faire des "disparitions" une priorité avec la publication de liste de "disparus". Vient s'ajouter la création de comités dans quarante villes du Maroc. Ces comités ont l'intention de faire de 1994 une année spécialement réservée aux disparitions et cette fois-ci Marocains et Sahraouis seront concernés. Quant au pouvoir, nous pouvons avoir quelques espoirs, même s'ils restent un peu mitigés :

- Amnesty International a pu se rendre à deux reprises au Maroc en 1993, ce qui n'était pas arrivé depuis 1990. Elle a même pu avoir des rencontres intéressantes avec des interlocuteurs concernés par les problèmes des Droits de l'Homme.

- En décembre dernier le Ministre de l'Intérieur et le Conseil Consultatif des Droits de l'Homme (créé par le roi) ont tous deux déclaré qu'ils étaient déterminés à liquider tous les dossiers relatifs au Droits de l'Homme. Néanmoins ils démentaient catégoriquement, une fois de plus, l'existence de centres secrets et donnaient 
l'assurance que des certificats de décès seraient envoyés aux familles. A ce sujet, nous ne savons que penser car, à ce jour, nous n'avons aucune nouvelle des 600 "disparus" pour lesquels nous avons remis une liste aux autorités marocaines.

- Un Ministre délégué auprès du Premier Ministre, chargé des Droits de l'Homme, a été nommé dans le nouveau gouvernement. Bien sûr, nous pouvons nous interroger : "A-til le désir d'agir et le laissera-t-on faire ou est-ce une nouvelle façade et un comportement caricatural du pouvoir marocain vis-à-vis des ses engagements internationaux? A l'extérieur, malgré quelques interventions du Département d'Etat américain ou du Parlement Européen, dénonçant même fermement les violations des Droits de l'Homme, le Maroc donne l'image d'un pays stable et sûr à l'étranger, comparé à la situation actuelle en Algérie. Ceci est notamment dû aux bonnes relations économiques avec les pays occidentaux y compris ceux d'Amérique du Nord.

\section{NOTES}

1. Résolution 47/133. Articles 1,2 et 7.

2. AI considère que ces deux expressions sont presque des synonymes.

3. Rapport du Groupe de travail sur les disparitions forcées ou involontaires, E/CN. 4/1993/25, 7 janvier 1993, Conseil Economique et Social, ONU, p. 137.

4. Rapport 1993, Editions Francophones d'Amnesty International, Paris, 1993.

5. Cette liste ne concerne que l'année 1992, la situation des droits de l'homme pouvant être extrêmement mouvante. Elle n'a pas un caractère exhaustif, ni pour ce qui concerne les pays, ni pour ce qui concerne les violations énumérées. Pour chaque pays, l'ordre de présentation des violations n'a pas de signification particulière.

6. Exécutions extrajudiciaires.

7. "Where have all the prisoners gone ?" in Index on Censorship, 10/93, p.4.

8. Pour Amnesty, les prisonniers d'opinion sont détenus du fait de leurs convictions politiques ou religieuses ou pour toute autre raison de conscience ou du fait de leur origine ethnique, de leur sexe, de leur couleur ou de leur langue et n'ont ni usé de violence ni préconisé son usage (Article 1, alinéa a des Statuts internationaux de l'organisation).

9. Amnesty International, Reply to "Where have all the prisoners gone ?", Londres, mars 1994.

10. François Bédarida,"Dans les ténèbres du IIIème Reich - Nuit et Brouillard", in La disparition, Editions Actes Sud, à paraître.

11. Voir Annexe Maroc.

12. Il convient de noter que la définition internationale des "disparitions" fait intervenir parmi les critères déterminants celui de l'implication gouvernementale. Sur le strict plan juridique, de tels actes par des groupes d'opposition armés ne seraient donc pas qualifiés de "disparitions". Voir à ce sujet le préambule de la Déclaration sur la protection de toutes les personnes contre les disparitions forcées (ONU, 18 décembre 1992). En 1992, Amnesty International signale des "disparitions" de civils vietnamiens 
au Cambodge par la partie du Kampuchea Démocratique (Khmers Rouges). Cf. Rapport 1993, EFAI, 1993, p. 80-81.

13. Les Etats sont à la fois les auteurs et les sujets du droit international des droits de l'homme.

14. Cette expression doit être prise dans un sens large et vise à les distinguer d'organisations criminelles.

15. Sous réserve de la définition juridique évoquée dans la note 12.

16. Amnesty international.

INDEX

Mots-clés : droits de l'Homme, disparitions

\section{AUTEUR}

\section{FRANCIS PERRIN}

Cet article se fonde sur les nombreux documents et rapports d'Amnesty International qui ont décrit le phénomène des disparitions forcées. Citons, en particulier : Les disparus, Editions Francophones d'Amnesty International - Seuil, Paris, 1981 ; "Disparitions" et assassinats politiques dans les années 80-90. L'inacceptable, Editions Francophones d'Amnesty International, Paris, 1993 ; "Disappearances" and political killings. Human rights crisis of the 1990s". Manual for action. Amnesty International Dutch section, Amsterdam, 1994. Les conclusions de cet article n'engagent cependant que leur auteur. 